PROCEEDINGS OF THE

AMERICAN MATHEMATICAL SOCIETY

Volume 134, Number 3, Pages 749-753

S 0002-9939(05)07991-8

Article electronically published on July 18, 2005

\title{
ON THE NON-STARLIKENESS OF SOLUTIONS TO THE STARLIKE INTERIOR WAKE PROBLEM
}

\author{
ANDREW ACKER
}

(Communicated by Richard A. Wentworth)

\begin{abstract}
We study examples of the starlike interior "wake problem" for which no starlike solution exists relative to the natural star center of the problem. These examples show that the main result of D.E. Tepper in "A mathematical model for a wake" (Michigan Math. J. 31 (1984), 161-165) is not correct.
\end{abstract}

\section{Introduction AND MAin RESUlts}

We study the 2-dimensional "finite interior wake problem" in the form studied by D.E. Tepper [T2. Here, an ideal fluid inside a closed 2-dimensional vessel circulates around an obstacle in the vessel. The flow may have free surfaces, due to separation of the flow from the obstacle (called cavitation). The mathematical problem is as follows:

1.1. Interior wake problem. In the plane $\Re^{2}$, let the nested, bounded domains $G$ and $H$ be given with $\mathrm{Cl}(H) \subset G$. For any $\lambda>0$, we seek a simple closed curve $\Gamma_{\lambda} \subset G \backslash H$ such that

$$
\begin{gathered}
\left|\nabla U_{\lambda}\right|=\lambda \quad \text { on } \quad \Gamma_{\lambda} \backslash(\partial H), \\
\liminf _{\Omega_{\lambda} \ni q \rightarrow p}\left|\nabla U_{\lambda}(q)\right| \geq \lambda \quad \text { for all } p \in \Gamma_{\lambda},
\end{gathered}
$$

where $U_{\lambda}(p)$ denotes the capacitary potential in the annular domain $\Omega_{\lambda}$ bounded by $\Gamma_{\lambda} \cup \partial G$ (thus $U_{\lambda}$ solves the Dirichlet problem: $\Delta U_{\lambda}=0$ in $\Omega_{\lambda}, U_{\lambda}\left(\Gamma_{\lambda}\right)=0$, $\left.U_{\lambda}(\partial G)=1\right)$.

Tepper's study of Problem 1.1 in T2 is restricted to the starlike case. His main result states that if $G$ and $H$ are starlike domains having the same star center, then for $\lambda$ sufficiently large, Problem 1.1 has at least one solution $\Gamma_{\lambda}$, which is also starlike relative to the same star center. (The same claim appears in [T3, Theorem 1], with reference to [T2.)

We will show that this claim is false: that in general the starlike interior wake problem does not have any solution which is starlike relative to the common star center of the two given boundaries (see Theorem 1). We will discuss the source and

Received by the editors July 1, 2004 and, in revised form, October 8, 2004.

2000 Mathematics Subject Classification. Primary 35R35, 76B07.

Key words and phrases. Ideal fluid, free boundary, geometric constraint, cavitation, starlikeness. 
consequences of Tepper's error in the concluding remarks. It's worth observing that Tepper's claim is not entirely unreasonable, or it would not have stood unchallenged in print for 20 years. In fact the corresponding claim for the exterior wake problem (see [T3]) is true.

Theorem 1. Assume in Problem 1.1 that $G$ is a starlike $C^{2}$-domain having the origin 0 as its star center, and that $\partial G$ contains a radial line segment. Then no solution $\Gamma_{\lambda}$ at $\lambda>0$ can be starlike relative to 0 if $\lambda$ is sufficiently large.

\section{Proof of Theorem 1}

Step 1. Under the assumptions of Theorem 1, we have

$$
\operatorname{dist}(p, \partial G) \leq(C / \lambda)
$$

for all sufficiently large $\lambda>0$, all solutions $\Gamma_{\lambda}$ of Problem 1.1 at $\lambda$, and all points $p \in \Gamma_{\lambda}$. Thus $(\partial H) \cap \Gamma_{\lambda}=\emptyset$ for sufficiently large $\lambda>0$.

The proof follows by minor adjustments in the proof of [A4, Lemma 2.5].

Step 2. Under the assumptions of Theorem 1, let $\Gamma_{\lambda}$ denote a solution at $\lambda>0$. Then there exist constants $M$ and $\lambda_{0}>0$ such that for any $\lambda \geq \lambda_{0}$, the function $\phi_{\lambda}(p):=\operatorname{Ln}\left(\left|\nabla U_{\lambda}(p)\right| / \lambda\right)$ is such that

$$
\left|\phi_{\lambda}(p)\right| \leq M \quad \text { in } \quad \Omega_{\lambda} .
$$

Proof. Let $r_{0}=1 / K_{0}$, where $K_{0}=\max \{|K(p)|: p \in \partial G\}$ and $K(p)$ denotes the curvature of $\partial G$ at $p \in \partial G$. Using Step 1, choose $\lambda_{0}>0$ so large that $\sup \left\{\operatorname{dist}(p, \partial G): p \in \Gamma_{\lambda}\right\}<\min \left\{\left(r_{0} / 2\right)\right.$, $\left.\operatorname{dist}(\partial G, \partial H)\right\}$ (thus $\left|\nabla U_{\lambda}(p)\right|=\lambda$ for all $\lambda>\lambda_{0}$ and for all $p \in \Gamma_{\lambda}$ ). Given a point $p_{0} \in \partial G$, choose the points $p_{0}^{ \pm}$(with $p_{0}^{-} \in G$ and $\left.p_{0}^{+} \in \Re^{2} \backslash G\right)$ such that $B\left(p_{0}^{ \pm} ; r_{0}\right)$ are the interior and exterior tangent balls (of radius $r_{0}$ ) to $\partial G$ at $p_{0}$. Set $\omega_{\lambda}^{ \pm}:=\left\{0<u_{\lambda}^{ \pm}(p)<1\right\}$ and $\gamma_{\lambda}^{ \pm}:=\left\{u_{\lambda}^{ \pm}(p)=0\right\}$, where

$$
u_{\lambda}^{ \pm}(p)=\operatorname{Ln}\left(\left|p-p_{0}^{ \pm}\right| / \alpha_{\lambda}^{ \pm}\right) / \operatorname{Ln}\left(r_{0} / \alpha_{\lambda}^{ \pm}\right)
$$

and $\alpha_{\lambda}^{ \pm}:=\operatorname{dist}\left(p_{0}^{ \pm}, \Gamma_{\lambda}\right)$. It is easily seen that $\gamma_{\lambda}^{-} \subset G \backslash \Omega_{\lambda}, \gamma_{\lambda}^{+} \cap\left(G \backslash \Omega_{\lambda}\right)=\emptyset$, and $\Gamma_{\lambda} \cap \gamma_{\lambda}^{ \pm} \neq \emptyset$. We also have that

$$
\pm\left(u_{\lambda}^{ \pm}(p)-U_{\lambda}(p)\right) \leq 0
$$

on $\partial\left(\omega_{\lambda}^{ \pm} \cap \Omega_{\lambda}\right)$, and therefore throughout the domains $\left(\omega_{\lambda}^{ \pm} \cap \Omega_{\lambda}\right)$, by the maximum principle for harmonic functions. Therefore, we have that

$$
\begin{gathered}
\pm\left|\nabla U_{\lambda}\left(p_{0}\right)\right| \leq \pm\left|\nabla u_{\lambda}^{ \pm}\left(p_{0}\right)\right|=1 /\left(r_{0} \operatorname{Ln}\left(r_{0} / \alpha_{\lambda}^{ \pm}\right)\right)=\left(\alpha_{\lambda}^{ \pm} / r_{0}\right)\left(1 /\left(\alpha_{\lambda}^{ \pm} \operatorname{Ln}\left(r_{0} / \alpha_{\lambda}^{ \pm}\right)\right)\right) \\
= \pm\left(\alpha_{\lambda}^{ \pm} / r_{0}\right)\left|\nabla u_{\lambda}^{ \pm}\left(p^{ \pm}\right)\right| \leq \pm\left(\alpha_{\lambda}^{ \pm} / r_{0}\right)\left|\nabla U_{\lambda}\left(p^{ \pm}\right)\right|= \pm\left(\lambda \alpha_{\lambda}^{ \pm} / r_{0}\right)
\end{gathered}
$$

for any points $p^{ \pm} \in \Gamma_{\lambda} \cap \gamma_{\lambda}^{ \pm}$, where $\left(r_{0} / 2\right) \leq \alpha_{\lambda}^{ \pm} \leq\left(3 r_{0} / 2\right)$ independent of $p_{0} \in \partial G$ and $\lambda>\lambda_{0}$. Therefore, we have

$$
\operatorname{Ln}(1 / 2) \leq \phi_{\lambda}(p) \leq \operatorname{Ln}(3 / 2)
$$

on $\partial \Omega_{\lambda}=\partial G \cup \Gamma_{\lambda}$, and therefore throughout $\Omega_{\lambda}$, by the maximum principle for harmonic functions. The assertion follows.

Step 3. Under the assumptions of Theorem 1, let $L=\left[p_{1}, p_{2}\right]$ denote a straight line segment in $\partial G$ containing its (distinct) endpoints $p_{1}, p_{2}$, and let $L_{0}$ denote the corresponding open line segment. For any fixed closed sub-segment $I=\left[q_{1}, q_{2}\right]$ of $L_{0}$, let $S(I)=\left\{p \in \Re^{2}: \tau \cdot q_{1}<\tau \cdot p<\tau \cdot q_{2}\right\}$, where $\tau=\left(\left(p_{2}-p_{1}\right) /\left|p_{2}-p_{1}\right|\right)$. 
For large $\lambda>0$, let $\Gamma_{\lambda}$ solve Problem 1.1 at $\lambda$, and let $\Omega_{\lambda}(I)$ denote the connected component of $\Omega_{\lambda} \cap S(I)$ whose boundary intersects $I$. Then there exist uniform constants $A, B>0$ such that

$$
\left|\phi_{\lambda}(p)\right| \leq A \cdot \operatorname{Exp}(-B \lambda)
$$

in $\Omega_{\lambda}(I)$ for all sufficiently large $\lambda>0$.

Proof. Choose local Cartesian coordinates $(x, y)$ such that $L=[0, \alpha] \times\{0\}$ for $\alpha:=$ $\left|p_{2}-p_{1}\right|>0$ and $[0, \alpha] \times(0, \beta] \subset G$ for sufficiently small $\beta>0$. Set $R_{\lambda}(L)=(0, \alpha) \times$ $(0, \beta(\lambda))$, where $\beta(\lambda)>0$ is as small as possible consistent with the requirement that $\Omega_{\lambda}(L) \subset R_{\lambda}(L)$. Observe that $\Delta \phi_{\lambda}=0$ in $\Omega_{\lambda},\left|\phi_{\lambda}\right| \leq M$ in $\Omega_{\lambda}$ (by Step 2), $\phi_{\lambda}\left(\Gamma_{\lambda}\right)=0$, and $\partial \phi_{\lambda} / \partial y=-\partial \operatorname{Arg}\left(\nabla U_{\lambda}\right) / \partial x=0$ on $L$. Assume $I:=[\delta, \alpha-\delta] \times\{0\}$ for small $\delta>0$. For any $x_{0} \in[\delta, \alpha-\delta]$, we conclude by comparison principles, including the Hopf boundary point lemma, that

$$
\pm \phi_{\lambda}(x, y) \leq \psi_{\lambda}\left(x_{0} ; x, y\right):=C_{0} \cosh \left(\pi\left(x-x_{0}\right) / 4 \beta\right) \cos (\pi y / 4 \beta),
$$

throughout the intersection of $\Omega_{\lambda}(L)$ with $\left[x_{0}-\delta, x_{0}+\delta\right] \times(0, \beta)$, where $C_{0}:=$ $(2 M / \cosh (\pi \delta / 4 \beta))$ and $\beta=\beta(\lambda)$. It follows that $\left|\phi_{\lambda}(p)\right| \leq(2 M / \cosh (\pi \delta / 4 \beta))$ throughout $\Omega_{\lambda}(I)$. The assertion follows from this, in view of the fact that $0<$ $\beta(\lambda) \leq(C / \lambda)$ (by Step 1$)$.

Step 4. In the context of Step 3, there exist a value $\lambda_{0}>0$ and a null function $z(\cdot)$ such that for any $\lambda \geq \lambda_{0}$ and any point $p_{\lambda} \in \Gamma_{\lambda}(I):=\Gamma_{\lambda} \cap\left(\partial \Omega_{\lambda}(I)\right)$, we have that

$$
\begin{gathered}
\operatorname{dist}\left(p_{\lambda}, \partial G\right)=\operatorname{dist}\left(p_{\lambda}, L_{0}\right), \\
\left|\operatorname{dist}\left(p_{\lambda}, L_{0}\right)-(1 / \lambda)\right| \leq(1 / \lambda) z(1 / \lambda)
\end{gathered}
$$

as $\lambda \rightarrow \infty$.

Proof. We remark that $z(t)$ is a null function if $z(t) \rightarrow 0$ as $t \rightarrow 0+$. The first assertion, (3), follows easily from Step 1. Turning to the proof of (4), we choose a closed segment $J$ such that $I \subset J_{0} \subset J \subset L_{0}$. For given $p_{\lambda} \in \Gamma_{\lambda}(I)$, let $\gamma_{\lambda}$ denote the curve of steepest ascent of $U_{\lambda}$ joining $p_{\lambda}$ to $\partial G$. Since $\left|\nabla U_{\lambda}\right| \geq C \lambda$ in $\Omega_{\lambda}$ by Step 2 (where $C>0$ is independent of large $\lambda$ ), we have

$$
\left|\gamma_{\lambda}\right| \leq \int_{\gamma_{\lambda}}\left(\left|\nabla U_{\lambda}\right| / C \lambda\right) d s=(1 / C \lambda)
$$

where $\left|\gamma_{\lambda}\right|$ denotes the Euclidean arc length of $\gamma_{\lambda}$. Therefore, for any $\delta>0$, we have $\left|\gamma_{\lambda}\right|<\delta$ for sufficiently large $\lambda>0$, from which it follows for all sufficiently large $\lambda>0$ that $\gamma_{\lambda} \subset \Omega_{\lambda}(J)$. By Step 3, there is a null function $z(\cdot)$ such that

$$
\max \left\{|| \nabla U_{\lambda}(p)|-\lambda|: p \in \Omega_{\lambda}(J)\right\} \leq z(1 / \lambda)
$$

as $\lambda \rightarrow \infty$. It follows from (5) (and the fact that $\gamma_{\lambda} \subset \Omega_{\lambda}(J)$ ) that

$$
(\lambda-z(1 / \lambda)) \operatorname{dist}\left(p_{\lambda}, J\right) \leq(\lambda-z(1 / \lambda))\left|\gamma_{\lambda}\right| \leq \int_{\gamma_{\lambda}}\left|\nabla U_{\lambda}\right| d s=1
$$

for a null function $z(\cdot)$. Now, again given a point $p_{\lambda} \in \Gamma_{\lambda}(I)$, choose $q_{\lambda} \in J$ such that $\left|p_{\lambda}-q_{\lambda}\right|=\operatorname{dist}\left(p_{\lambda}, J\right)$, and let $\gamma_{\lambda}$ now denote the straight line segment joining 
$p_{\lambda}$ to $q_{\lambda}$. Then

$$
\begin{gathered}
1=\int_{\gamma_{\lambda}}\left(d U_{\lambda} / d s\right) d s \leq \int_{\gamma_{\lambda}}\left|\nabla U_{\lambda}\right| d s \\
\leq(\lambda+z(1 / \lambda))\left|\gamma_{\lambda}\right|=(\lambda+z(1 / \lambda)) \operatorname{dist}\left(p_{\lambda}, J\right)
\end{gathered}
$$

for some null function $z(\cdot)$, again due to (5) and the fact that $\gamma_{\lambda} \subset \Omega_{\lambda}(J)$. The assertion (4) follows from (6) and (7).

Step 5. Proof of Theorem 1. If $\Gamma_{\lambda}$ solves Problem 1.1, then $(\partial H) \cap \Gamma_{\lambda}=\emptyset$ if $\lambda$ is sufficiently large, by Step 1 . Let $L$ be a radial line segment in $\partial G$. Choose points $p_{0}, q_{0} \in L_{0}$ such that $\left|p_{0}\right|<\left|q_{0}\right|$. For any sufficiently large $\lambda>0$, choose the points $p_{\lambda}, q_{\lambda} \in \Gamma_{\lambda}$ such that $p_{\lambda}=p_{0}+\alpha_{\lambda} \nu$ and $q_{\lambda}=q_{0}+\beta_{\lambda} \nu$, where $\alpha_{\lambda}, \beta_{\lambda}>0$ and $\nu$ denotes the interior normal to $\partial G$ on $L_{0}$. In order for $\Gamma_{\lambda}$ to be starlike relative to the origin, it is necessary for the line segment joining $q_{\lambda}$ to the origin to be contained in $\mathrm{Cl}\left(\Omega_{\lambda}\right)$, which implies that $\beta_{\lambda} \geq\left(\left|q_{0}\right| /\left|p_{0}\right|\right) \alpha_{\lambda}$. It follows by double application of (4) that

$$
(1 / \lambda)(1+z(1 / \lambda)) \geq \beta_{\lambda} \geq\left(\left|q_{0}\right| /\left|p_{0}\right|\right) \alpha_{\lambda} \geq\left(\left|q_{0}\right| /\left|p_{0}\right|\right)(1 / \lambda)(1-z(1 / \lambda)) .
$$

This is clearly false for sufficiently large $\lambda$. Therefore, the assertion holds.

\section{Concluding REMARKS}

Remark 1 (Tepper's error in T2]). In his existence proof in [T2, Tepper's method is to treat Problem 1.1 as the limiting case of a sequence of "soft-barrier" problems. A soft-barrier problem is a variant of Problem 1.1 in which the conditions (1) and (2) are replaced by the single requirement that $|\nabla U(p)|=Q(p)$ on the free boundary $\Gamma$, for a given, strictly-positive continuous function $Q(p): \Re^{2} \rightarrow \Re_{+}$. In his Lemma 3 , Tepper states correctly (with reference to the present author [A1) that if the function $\phi(\lambda):=\lambda Q(\lambda p)$ is weakly decreasing in $\lambda>0$ for any fixed $p \in \Re^{2}$ (where 0 is the star center), then the soft-barrier problem has at most one solution, and that solution must be starlike and contains no radial line segments. Tepper obtains (the interior complement of) his solution as the union of an infinite collection of (interior complements of) soft-barrier solutions. The problem is that Tepper's "soft barrier" functions $Q_{n}(p)$ do not satisfy the conditions of his Lemma 3 . Then the rest of the proof fails, since his soft barrier problems do not necessarily have starlike solutions, as follows as a further consequence of the present counterexample.

Remark 2 (comments on [T3]). In [T3, Tepper treats a double-free-boundary problem involving geometric constraints (hard barriers) on both free boundaries. Tepper's proof of his main result, T3, Theorem 2], is based on an iterative scheme involving alternate starlike solutions of the interior and exterior wake problems. The proof is not valid because it depends in a crucial way on the main result in T2] (although Tepper's analogous result for the exterior wake problem is correct). Essentially the same existence result, but without the claims of starlikeness, appears in The Collected Works of A. Beurling [B2] (1989), Chapter 6: A free boundary problem in the annulus (see Main Theorem, p. 434). It is easy to see that Beurling's solution is in fact starlike under Tepper's assumptions, so that Tepper's claim in [T3. Theorem 2] is valid. We remark that Tepper's argument is essentially a reduction of Beurling's argument to the starlike case. The present author also studied 
the double-free-boundary problem with geometric constraints, but in a slightly different geometric context (see [A2]). The author's existence results followed in the limiting case from a study of double-free-boundary soft-barrier problems (see A3]).

Remark 3 (Tepper's wake and nozzle flow-models). Tepper very briefly generalizes his results in T2 and T3 to the case of fluid flows in infinite domains. Thus, Problem 1.1 generalizes to the case of a flow around an obstacle $H$ in an infinite strip-like stream bed of the form $G:=\left\{\left(p=(x, y) \in \Re^{2}: \phi_{1}(x)<y<\phi_{2}(x)\right\}\right.$. It is important to realize that, contrary to the impression suggested by the infinitestream-bed geometry, the flow in this model is restricted to local circulation around the obstacle $H$, with no net flow down the stream. The nozzle problem in [T3] (flow through a nozzle with an inner "obstacle") is also restricted to the case of pure circulation around the obstacle (i.e., the fluid flows out of the nozzle on one side of the obstacle and back in on the other side).

\section{REFERENCES}

[A1] A. ACKER: Heat flow inequalities with application to heat flow optimization problems. SIAM J. Math. Anal 8(1977), pp. 604-618. MR0473960 (57:13618)

[A2] A. ACKer: Some free boundary optimization problems and their solutions. Numerische Behandlung von Differentialgleichungen mit besonderer Berucksichtigung freier Randwertaufgaben (J. Albrecht, L. Collatz, G. Hammerlin, editors), Birkhäuser Verlag, Basel 1978, pp. 9-22. (Proceedings of the conference by the same name held at the Mathematisches Forschungsinstitut Oberwolfach, Germany, May 1-7, 1977.) MR0497989 (80m:35078)

[A3] A. ACKer: A free boundary optimization problem involving weighted areas, J. Appl. Math. and Phys. (ZAMP) 29 (1978), pp. 395-408. MR0482457 (58:2524a)

[A4] A. ACKER: On the qualitative theory of parametrized families of free boundaries. J. reine angew. Math. 193(1989), pp. 134-167. MR0972364 (90a:35229)

[A5] A. ACKER: Uniqueness and monotonicity of solutions for the interior Bernoulli free boundary problem in the convex, $n$-dimensional case. Nonlinear Analysis, TMA 13(1988), pp. 14091425. MR.1028238 (91a:35168)

[B1] A. Beurling: On free boundary problems for the Laplace equation. Seminars on Analytic Functions, Vol. I (1957), pp. 248-263. Institute for Advanced Study, Princeton, N.J.

[B2] A. Beurling: The Collected Works of Arne Beurling, Vol. 1. (edited by Carleson, L., Malliavan, P., Neuberger, J., and Werner, J.), Birkhäuser Boston, 1989. MR.1057613 (92k:01046a)

[T1] D.E. Tepper: A free boundary problem in the annulus. J. Austral. Math. Soc. (Series A) 34(1983), pp. 177-181. MR0687323(84c:31001)

[T2] D.E. Tepper: A mathematical model for a wake. Mich. Math. J. 31(1984), pp. 161-165. MR0752252 (86b:35205)

[T3] D.E. Tepper: A jet around an obstacle. In: Topics in Complex Analysis (Proceedings of the special session held at Fairfield, Conn., Oct. 28-29, 1983, D. Shaffer, editor). Contemporary Mathematics series, Vol. 38, American Mathematical Society, 1985, pp. 127-132. MR0789455

Department of Mathematics and Statistics, Wichita State University, Wichita, KANSAS 67208-0033

E-mail address: acker@math.wichita.edu 